\section{Aortic Aneurysm and Pseudoaneurysm Assessment}

Although contrast angiography is still considered the "gold standard" for the evaluation of the aorta and its major branches, magnetic resonance angiography (MRA) has quickly gained popularity as an imaging tool for the assessment of the entire aorta. MRA serves as an alternative imaging modality that can be utilized in patients with impaired renal function and with allergies to iodinated contrast medium (iodinated contrast medium is required in contrast angiography and computed tomography, CT). The purpose of this unit is to present fundamental MRA techniques useful in the evaluation of the thoracic and abdominal aorta based on experience on a 1.5 T GE LX scanner.

\section{IMAGING OF THORACIC AORTA}

The sequences used in the evaluation of the thoracic aorta include black-blood $T_{1}$ weighted or $T_{2}$-weighted images as well as white-blood gadolinium-enhanced short $T_{\mathrm{R}}$ gradient echo images. For the black-blood sequence, the authors use cardiac-gated double inversion recovery fast spin echo imaging in the transverse plane. However, cardiac-gated $T_{1}$-weighted transverse spin echo images are an acceptable alternative. The authors perform gadolinium-enhanced imaging as a standard dynamic 3-D acquisition in the sagittal oblique plane. The entire Basic Protocol 1 will take $\sim 30$ min to perform.

Table A12.2.1 outlines the equipment parameters necessary for imaging of the thoracic aorta.

NOTE: Be sure that technologists and nurses have immediate access to any emergency equipment that may be relevant to a given study, or that may be needed for a particular patient, such as crash carts or oxygen.

\section{Materials}

Normal saline $(0.9 \% \mathrm{NaCl})$, sterile

Gadolinium-based MR contrast agent (e.g., Magnevist, Omniscan, or Prohance)

\section{Set up patient and equipment}

1. Screen patients for ferromagnetic materials, such as cardiac pacemakers, cochlear implants, and intracranial aneurysm clips, which preclude them from MR imaging. Know the patient's medical and surgical history, such as respiratory or cardiac conditions or presence of spinal hardware, which is critical to optimize the patient's MRI exam.

Generally, standard screening forms (APPENDIX 1) are used for all patients scanned in a magnetic resonance system.

Table A12.2.1 Equipment Parameters for Thoracic Aorta Imaging

\begin{tabular}{ll}
\hline Coil type & $\begin{array}{l}\text { Body coil } \\
\text { Gradient coil strength }\end{array}$ \\
$\begin{array}{l}\text { required } \\
\text { Cardiac gating }\end{array}$ & Yes \\
Peripheral gating & No \\
Respiratory gating & Yes \\
Oxygen & 2-liter nasal cannula if required by patient \\
Use of contrast agents & Yes, $40 \mathrm{ml}$ gadolinium chelate i.v. \\
\hline
\end{tabular}

Contributed by Maricela Contreras, Rajesh S. Amin, and E. Kent Yucel

Current Protocols in Magnetic Resonance Imaging (2001) A12.2.1-A12.2.14

Copyright $\odot 2001$ by John Wiley \& Sons, Inc.
UNIT A12.2

BASIC

PROTOCOL 1

Acquired Aortic

Disease

A12.2.1

Supplement 3 
The presence of any ferromagnetic metals may be a health hazard to the patient when he or she is inside the magnet, and will also affect the imaging. If in doubt as to the exact composition of the items, it is best to exclude patients with any metal implants; see Shellock (1996) for discussion of what implants may be safely scanned using magnetic resonance.

Patients may be accompanied into the magnet room by a friend or family member, who can sit in the room during the scan and comfort the patient as needed. This companion must be screened as well to ensure the absence of loose metal objects on the body or clothing.

2. If the procedure is a research protocol, have the patient sign any necessary consent form.

3. Have the patient remove all jewelry and change into a gown to eliminate any metal that might be found in clothing.

4. Have the patient wash off any mascara and other makeup to avoid local tissue heating and image artifacts.

5. Inform the patient about what will occur during the procedure, what he or she will experience while in the magnet, and how to behave, including the following:

a. If earphones or headphones are used to protect the ears from the loud sounds produced by the gradients, the patient will be asked to wear these, but will be able to communicate with you at any time during the imaging.

b. The patient will be given a safety squeeze-bulb or similar equipment to request assistance at any time.

c. For good results the patient should not talk, and should avoid or minimize swallowing or other movement, during each scan, i.e., as long as the banging sounds continue. Between scans, talking and swallowing are allowed in most cases, but should be avoided when comparative positional studies are being performed; the patient will be informed when this is the case.

d. Nevertheless, the patient may call out at any time if he or she feels it necessary.

6. Instruct the patient how to hold his or her breath at end expiration for 20 to $30 \mathrm{sec}$. Supplemental oxygen may facilitate breath-holding. Anxiolytics, such as Valium or Xanax, may be useful in patients who are anxious or claustrophobic.

7. Have the patient mount onto the table in the supine position with feet first in the magnet. Either before or right after the patient lies down, set up any triggering devices or other monitoring equipment that are to be used.

8. Place electrocardiogram (ECG) leads on the patient in accordance with need.

9. Place a 20- to 22-G i.v. catheter, preferably in an antecubital vein, for the injection of gadolinium-based MR contrast agent during imaging. The patient's arms are positioned above his or her head to decrease wraparound artifact.

10. If needed, place a pillow or other support under the knees to make the patient more comfortable.

11. Use the centering light to position the patient and put him or her into the center of the magnet.

The patient's chest should be centered in the body coil. If only the thoracic aorta is to be imaged, the nipple line can be used as a landmark. Otherwise, the xyphoid can be used as an anatomical landmark if the entire aorta is to be imaged.

Aortic

Aneurysm and Pseudoaneurysm Assessment

\section{A12.2.2}

Supplement 3 
Table A12.2.2 Primary Clinical Imaging Parameters for Sequence 1 (Pilot Scan)

Patient position

Scan type

Imaging plane (orientation)

Central slice or volume center

Echo time $\left(T_{\mathrm{E}}\right)$

Repeat time $\left(T_{\mathrm{R}}\right)$

Flip angle (FA)

Fields of view $\left(\mathrm{FOV}_{x}, \mathrm{FOV}_{y}\right)$

Resolution $(\Delta x, \Delta y)$

Number of data points collected $\left(N_{\mathrm{x}}, N_{\mathrm{y}}\right)$

Display matrix $\left(D_{\mathrm{x}}, D_{\mathrm{y}}\right)$

Slice thickness $(\Delta z)$

Number of slices

Slice gap

Number of acquisitions $\left(N_{\text {acq }}\right)$

Swap read and phase encoding

Slice locations

Saturation pulses

Scan time
Supine, feet first

Short $T_{\mathrm{R}}$ gradient echo

Three planes (transverse, sagittal, and coronal)

Center of chest

$1.6 \mathrm{msec}$ (or minimum)

$8.1 \mathrm{msec}$ (or minimum)

$30^{\circ}$

$400 \mathrm{~mm}, 400 \mathrm{~mm}$

$1.56 \mathrm{~mm}, 3.13 \mathrm{~mm}$

256,128

256,256

$10 \mathrm{~mm}$

9/plane

$2 \mathrm{~mm}$

2

No

Variable

None

$57 \mathrm{sec}$

Table A12.2.3 Primary Clinical Imaging Parameters for Sequence 2 (Double Inversion Recovery Fast Spin Echo)

\begin{tabular}{ll}
\hline $\begin{array}{l}\text { Patient position } \\
\text { Scan type }\end{array}$ & $\begin{array}{l}\text { Supine, feet first } \\
\text { Double inversion recovery fast spin } \\
\text { echo }\end{array}$ \\
Imaging plane (orientation) & Transverse \\
Central slice or volume center & Center of chest \\
Echo time $\left(T_{\mathrm{E}}\right)$ & $40 \mathrm{msec}$ \\
Receiver bandwidth $(\mathrm{RBW})$ & $62.5 \mathrm{kHz}$ \\
Echo train length (ETL) & 32 \\
Repeat time $\left(T_{\mathrm{R}}\right)$ & $2 R$-to- $R$ intervals \\
Delay time $\left(T_{\mathrm{D}}\right)$ & Minimum $(\sim 12 \mathrm{msec})$ \\
Flip angle $(\mathrm{FA})$ & $90^{\circ}$ \\
Fields of view $(\mathrm{FOV}, \mathrm{FOV}, \mathrm{f})$ & $300 \mathrm{~mm}, 300 \mathrm{~mm}$ \\
Resolution $(\Delta x, \Delta y)$ & $1.17 \mathrm{~mm}, 1.56 \mathrm{~mm}$ \\
Number of data points collected $\left(N_{\mathrm{x}}, N_{\mathrm{y}}\right)$ & 256,192 \\
Display matrix $\left(D_{\mathrm{x}}, D_{\mathrm{y}}\right)$ & 512,512 \\
Slice thickness $(\Delta z)$ & $10 \mathrm{~mm}$ \\
Number of slices & $20($ or as needed $)$ \\
Slice gap & None \\
Number of acquisitions $\left(N_{\text {acq }}\right)$ & 2 \\
Swap read and phase encoding & No \\
Slice locations & Aortic arch to diaphragm \\
ZIP 512 & Yes \\
Slice series & Sequential \\
ECG gating & Yes \\
Scan time & 8 min, 8 sec \\
\hline
\end{tabular}

Acquired Aortic 
Table A12.2.4 Primary Clinical Imaging Parameters for Sequence 3 (Optional Cardiac-Gated Transverse $T_{1}$-Weighted Spin Echo for Black-Blood Imaging)

\begin{tabular}{ll} 
Patient position & Supine, feet first \\
Scan type & $T_{1}$-weighted spin echo \\
Imaging plane (orientation) & Transverse \\
Central slice or volume center & Center of chest \\
Echo time $\left(T_{\mathrm{E}}\right)$ & $20 \mathrm{msec}$ \\
Repeat time $\left(T_{\mathrm{R}}\right)$ & $\begin{array}{l}\text { Depends on heart rate }(1 R \text {-to- } R \\
\text { interval) }\end{array}$ \\
Delay time $\left(T_{\mathrm{D}}\right)$ & Minimum \\
Flip angle $(\mathrm{FA})$ & $90^{\circ}$ \\
Fields of view $\left(\mathrm{FOV}_{x}, \mathrm{FOV}_{y}\right)$ & $300 \mathrm{~mm}, 300 \mathrm{~mm}$ \\
Resolution $(\Delta x, \Delta y)$ & $1.17 \mathrm{~mm}, 1.88 \mathrm{~mm}$ \\
Number of data points collected $\left(N_{\mathrm{x}}, N_{\mathrm{y}}\right)$ & 256,160 \\
Display matrix $\left(D_{\mathrm{x}}, D_{\mathrm{y}}\right)$ & 256,256 \\
Slice thickness $(\Delta z)$ & $8 \mathrm{~mm}$ \\
Number of slices & 20 \\
Slice gap & $2 \mathrm{~mm}$ \\
Number of acquisitions $\left(N_{\text {acq }}\right)$ & 2 \\
Swap read and phase encoding & Yes \\
Read direction & Anterior to posterior \\
Slice locations & Aortic arch to diaphragm \\
Saturation pulses & None \\
Slice series & Sequential \\
ECG gating & Yes \\
Scan time & $\sim 4$ min, 36 sec \\
\hline
\end{tabular}

Once this step has been performed, so long as the patient does not move on the table, the table itself can be moved and then replaced in the same position as before without jeopardizing the positioning of one scan relative to another.

\section{Sequence 1: Three-plane localizer pilot scan}

12. Run sequence 1 according to Table A12.2.2.

Three-plane localizer pilot scan is obtained using a short $T_{R}$ gradient echo sequence. Patient may hold his or her breath in end expiration, if possible.

\section{Sequence 2: Transverse double inversion recovery fast spin echo (black-blood} sequence)

13. Instruct the patient breath normally. Run sequence 2 according to Table A12.2.3.

Double inversion recovery fast spin echo sequence is used for black-blood imaging and is implemented with cardiac gating and nonbreath-holding. Black-blood imaging enables accurate measurement of true aortic diameter, including patent and thrombosed portions of the lumen. Images are obtained as sequential slices with interpolation to 512 by 512 display matrix. The triggering window is $20 \%$ of the $R$-to-R interval with minimum delay after the $Q R S$ waves. Slice are obtained during free breathing.

\section{Sequence 3: Transverse $T_{1^{-}}$weighted spin echo (optional black-blood sequence)}

14. Run sequence 3 according to Table A12.2.4.

If patient is unable to hold his or her breath, the $T_{1}$-weighted transverse spin echo images can be obtained with cardiac gating and respiratory compensation. Images are sequentially acquired from the level of the diaphragm to the aortic arch. Also, the read direction is changed to anterior to posterior. 
Table A12.2.5 Primary Clinical Imaging Parameters for Sequence 4 (3-D Gadolinium-Enhanced Short $T_{\mathrm{R}}$ Gradient Echo)

\begin{tabular}{ll}
\hline Patient position & Supine, feet first \\
Scan type & 3-D short $T_{\mathrm{R}}$ gradient echo \\
Imaging plane (orientation) & Sagittal oblique \\
Central slice or volume center & Center of chest \\
Echo time $\left(T_{\mathrm{E}}\right)$ & About $\leq 3 \mathrm{msec}$ \\
Receiver bandwidth (RBW) & $31 \mathrm{kHz}$ \\
Repeat time $\left(T_{\mathrm{R}}\right)$ & About $\leq 10 \mathrm{msec}$ \\
Flip angle $(\mathrm{FA})$ & $35^{\circ}$ \\
Fields of view $\left(\mathrm{FOV}_{\mathrm{x}}, \mathrm{FOV}_{\mathrm{y}}\right)$ & $400 \mathrm{~mm}, 400 \mathrm{rmm}$, with $r=3 / 4$ \\
& $($ rectangular field of view) \\
Resolution $(\Delta x, \Delta y)$ & $1.56 \mathrm{~mm}, 1.56 \mathrm{~mm}$ \\
Number of data points collected $\left(N_{\mathrm{x}}, N_{\mathrm{y}}\right)$ & $256,256 r$, with $r=3 / 4($ rectangular \\
& field of view) \\
Display matrix $\left(D_{\mathrm{x}}, D_{\mathrm{y}}\right)$ & 512,512 \\
Slice thickness $(\Delta z)$ & $2.6 \mathrm{~mm}$ \\
Number of slices & 32 \\
Slice gap & None \\
Number of acquisitions $\left(N_{\mathrm{acq}}\right)$ & 0.5 (half Fourier) \\
Read direction & Superior to inferior \\
Slice locations & Aortic arch to diaphragm \\
ZIP 512 & Yes \\
ZIP 2 & Yes \\
Saturation pulses & Interleaved fat saturation pulse \\
Slice series & Sequential \\
ECG gating & No \\
Scan time & 38 sec
\end{tabular}

The number of slices acquired will depend on the heart rate. For example, for a heart rate of 75 beats per min, the number of slices acquired will be $\sim 24$ slices.

\section{Sequence 4: Sagittal oblique 3-D gadolinium-enhanced short $T_{R}$ gradient echo (infusion)}

15. Leave patient in the magnet and inject the gadolinium-based contrast agent, then flush the line with $10 \mathrm{ml}$ saline.

16. Determine the 3-D gadolinium-enhanced MRA volume position from the pilot scan. Begin the volume coverage 1 to $2 \mathrm{~cm}$ above the arch and extend caudally to the diaphragm. Use the aortic arch as the axis of the plane.

An automatic dose timing module such as Smartprep $(G E)$ or fluoroscopic triggering (Phillips), if available, can be used to track the bolus of gadolinium in the descending aorta.

17. Set injection rate of contrast agent at $2.5 \mathrm{ml} / \mathrm{sec}$ for a total of $40 \mathrm{ml}$.

A dose of $0.1 \mathrm{mmol} / \mathrm{kg}$ of contrast agent is usually given.

The authors use automatic triggering to detect the arrival of contrast agent in a region of interest in the aorta and to determine the time between injection and data acquisition for the central lines of the $k$-space (centric reordering). This allows for synchronization between the acquisition of central lines of $k$-space and the arterial phase of the exam.

If the reader wishes to run a test bolus sequence, see UNIT A10.1 or UNIT A12.1.

Acquired Aortic Disease

A12.2.5 
18. Start the injection of the contrast agent. When the bolus of gadolinium is detected in the aorta, instruct the patient to hold his or her breath. Wait $6 \mathrm{sec}$ and run sequence 4 according to Table A12.2.5.

The arterial phase images are obtained during the first breath-hold period.

An interleaved fat saturation pulse may be used to suppress background signal, if available. Spectrally fat-selective saturation pulses, such as SPECIAL (Spectral Inversion at Lipids, GE Medical Systems) can be used with a time of 22 msec before applying the rf pulse. Alternatively, a precontrast image may be obtained and mask subtraction performed.

In addition to acquiring sequential slices with interpolation to 512 by 512 display matrix in the plane of the slice, slices are reconstructed at half the interval in the slice select direction acquired.

19. Instruct the patient to breathe and take another breath-hold. Six seconds after the previous scan, run sequence 4 again to obtain the venous phase images.

BASIC PROTOCOL 2

Aortic

Aneurysm and Pseudoaneurysm Assessment

A12.2.6

\section{IMAGING OF ABDOMINAL AORTA}

For patients with suspected abdominal aortic aneurysm, transverse and sagittal blackblood images are necessary followed by coronal 3-D gadolinium-enhanced MRA on the abdominal aorta. The patient set-up for this exam is the same as in Basic Protocol 1. The equipment parameters (see Table A12.2.6) for abdominal aorta imaging are also similar, except no cardiac-gating is required. The entire Basic Protocol 2 will take $\sim 30 \mathrm{~min}$ to perform.

\section{Set up patient and equipment}

1. Repeat Basic Protocol 1, steps 1 to 11.

\section{Sequence 5: Three-plane localizer pilot scan}

2. Run three-plane localizer pilot scan using the short $T_{\mathrm{R}}$ gradient echo sequence according to Table A12.2.7. Patient may hold his or her breath in end expiration.

The body coil is used to ensure that both the aorta and iliac vessels are imaged. The pilot scan should include both kidneys and major vessels of the abdominal aorta (proximal celiac and superior mesenteric arteries, and renal arteries). In tall patients, the field of view may need to be increased to $48 \mathrm{~cm}$.

\section{Sequence 6: $T_{1}$-weighted spin echo (black-blood sequence)}

3. Run sequence 6 according to Table A12.2.8.

As in the thoracic aorta, transverse $T_{1}$-weighted spin echo images are acquired through the abdominal aorta for black-blood imaging. This allows accurate measurement of the aortic diameter and facilitates evaluation of the lumen for thrombus and patency. Respiratory compensation can also be utilized.

Table A12.2.6 Equipment Parameters for Abdominal Aorta Imaging

\begin{tabular}{ll}
\hline Coil type & Body coil \\
Gradient coil strength & At least $20 \mathrm{mT} / \mathrm{m}$ \\
Cardiac gating & No \\
Peripheral gating & No \\
Respiratory gating & Yes \\
Oxygen & 2 liter nasal cannula if required by patient \\
Use of contrast agents & Yes, $40 \mathrm{ml}$ gadolinium chelate \\
& intravenously \\
\hline
\end{tabular}


Table A12.2.7 Primary Clinical Imaging Parameters for Sequence 5 (Pilot Scan Abdominal Aorta)

Patient position

Scan type

Imaging plane (orientation)

Central slice or volume center

Echo time $\left(T_{\mathrm{E}}\right)$

Repeat time $\left(T_{\mathrm{R}}\right)$

Flip angle (FA)

Fields of view $\left(\mathrm{FOV}_{\mathrm{x}}, \mathrm{FOV}_{\mathrm{y}}\right)$

Resolution $(\Delta x, \Delta y)$

Number of data points collected $\left(N_{\mathrm{x}}, N_{\mathrm{y}}\right)$

Display matrix $\left(D_{\mathrm{x}}, D_{\mathrm{y}}\right)$

Slice thickness $(\Delta z)$

Number of slices

Slice gap

Number of acquisitions $\left(N_{\text {acq }}\right)$

Swap read and phase encoding

Slice locations

Saturation pulses

Scan time
Supine, feet first

Short $T_{\mathrm{R}}$ gradient echo

Three planes (transverse, sagittal, and coronal)

Center of abdomen (at level of umbilicus)

$1.6 \mathrm{msec}$

$8.1 \mathrm{msec}$

$30^{\circ}$

$400 \mathrm{~mm}, 400 \mathrm{~mm}$

$1.56 \mathrm{~mm}, 3.13 \mathrm{~mm}$

256,128

256,128

$10 \mathrm{~mm}$

9/plane

$2 \mathrm{~mm}$

2

No

Variable

None

$57 \mathrm{sec}$

Table A12.2.8 Primary Clinical Imaging Parameters for Sequence 6 ( $T_{1}$-Weighted Spin Echo)

Patient position

Scan type

Imaging plane (orientation)

Central slice or volume center

Echo time $\left(T_{\mathrm{E}}\right)$

Repeat time $\left(T_{\mathrm{R}}\right)$

Flip angle (FA)

Fields of view $\left(\mathrm{FOV}_{x}, \mathrm{FOV}_{y}\right)$

Resolution $(\Delta x, \Delta y)$

Number of data points collected $\left(N_{\mathrm{x}}, N_{\mathrm{y}}\right)$

Display matrix $\left(D_{\mathrm{x}}, D_{\mathrm{y}}\right)$

Slice thickness $(\Delta z)$

Number of slices

Slice gap

Number of acquisitions ( $N_{\text {acq }}$ )

Swap read and phase encoding

Scan time
Supine, feet first

$T_{1}$-weighted spin echo

Transverse

Center of abdomen (at level of umbilicus)

$20 \mathrm{msec}$

600-800 msec

$90^{\circ}$

$400 \mathrm{~mm}, 400 \mathrm{~mm}$

$1.56 \mathrm{~mm}, 2.50 \mathrm{~mm}$

256,160

256,256

$10 \mathrm{~mm}$

15-21

$2 \mathrm{~mm}$

2

No

-3-4 min
Acquired Aortic

Disease

A12.2.7 
Table A12.2.9 Primary Clinical Imaging Parameters for Sequence 7 (3-D Gadolinium-Enhanced Short $T_{\mathrm{R}}$ Gradient Echo)

\begin{tabular}{ll}
\hline Patient position & Supine, feet first \\
Scan type & 3-D short $T_{\mathrm{R}}$ Gradient Echo \\
Imaging plane (orientation) & Coronal \\
Central slice or volume center & $\begin{array}{l}\text { Center of abdomen (at level of } \\
\text { umbilicus) }\end{array}$ \\
Echo time $\left(T_{\mathrm{E}}\right)$ & About $\leq 3 \mathrm{msec}$ \\
Receiver bandwidth (RBW) & $62 \mathrm{kHz}$ \\
Repeat time $\left(T_{\mathrm{R}}\right)$ & About $\leq 10 \mathrm{msec}$ \\
Flip angle $(\mathrm{FA})$ & $35^{\circ}$ \\
Fields of view & $400 \mathrm{~mm}, 400 \mathrm{rmm}$, with $r=3 / 4$ \\
& $($ rectangular field of view) \\
Resolution $(\Delta x, \Delta y)$ & $1.56 \mathrm{~mm}, 2.34 \mathrm{~mm}$ \\
Number of data points collected $\left(N_{\mathrm{x}}, N_{\mathrm{y}}\right)$ & 256,128 \\
Display matrix $\left(D_{\mathrm{x}}, D_{\mathrm{y}}\right)$ & 512,512 \\
Slice thickness $(\Delta z)$ & $2.6 \mathrm{~mm}$ \\
Number of slices & 48 \\
Slice gap & None \\
Number of acquisitions $\left(N_{\text {acq }}\right)$ & 0.5 (half Fourier) \\
Swap read and phase encoding & No \\
Slice locations & Diaphragm to common femoral arteries \\
ZIP 512 & Yes \\
ZIP 2 & Yes \\
Saturation pulses & Interleaved fat saturation pulse \\
Slice series & Sequential \\
Scan time & 38 sec \\
\hline
\end{tabular}

4. Choose the imaging plane (orientation) to be sagittal. Run sequence 6 again according to the rest parameters in Table A12.2.8.

\section{Sequence 7: Coronal 3-D gadolinium-enhanced short $T_{R}$ gradient echo (infusion)}

The coronal 3-D gadolinium-enhanced short $T_{\mathrm{R}}$ gradient echo sequence is the primary sequence for demonstrating the entire abdominal aorta, its major vessels and the iliac arteries. The 3-D MRA volume position is determined from the pilot scan. The volume position must be anterior enough to include the origins of the celiac and superior mesenteric arteries and the common femoral arteries, if possible. The posterior border of the 3-D volume should include both iliac arteries, renal arteries, and kidneys.

For optimal timing of the gadolinium bolus in the abdominal aorta, a Smartprep or similar contrast timing module can be used. This is especially crucial during the arterial phase of the bolus. In the presence of an abdominal aortic aneurysm, a delay of $10 \mathrm{sec}$ can be used with the contrast timing module. Otherwise, a delay of 5 or $6 \mathrm{sec}$ will suffice if the aorta is of normal caliber. The gadolinium injection rate for the aorta should be $2.5 \mathrm{ml} / \mathrm{sec}$ for a total of $40 \mathrm{ml}$.

Images are acquired in the arterial and venous phases using the method as described in Basic Protocol 1, steps 18 and 19.

Aortic

Aneurysm and Pseudoaneurysm Assessment

A12.2.8

5. Repeat Basic Protocol 1, step 15. Determine the slice positions as described above. Repeat Basic Protocol 1, step 17. 
6. Start the injector (contrast timing module). After $10 \mathrm{sec}$ (see above), instruct the patient to hold his or her breath and run sequence 7 according to Table A12.2.9.

An interleaved fat saturation pulse may be used to suppress background signal.

Images are also obtained as sequential slices with interpolation to a 512 by 512 display matrix. Slices are also reconstructed at half the interval (or l.3-mm increments).

\section{ANEURYSMS FOLLOW-UP}

Generally, the initial assessment of true and false aneurysms in patients with no contraindications to iodinated i.v. contrast can be performed with CT. However, in the presence of a contraindication or in the follow-up assessment of aortic aneurysms, MRI can be a useful alternative. In the situation of follow-up assessment, once the aneurysm has been thoroughly evaluated, MRI utilizing a black-blood technique, such as gradient echo sequences (FLASH, fast low angle shot-type imaging) or DIR (double inversion recovery) sequences in the transverse plane, can provide adequate imaging to monitor for size variations in a given interval time. Although contrast enhanced MRA is advantageous in the initial assessment of aneurysms, imaging without contrast would be more cost-effective in the follow-up evaluation.

\section{Repeat Basic Protocol 1, steps 1 to 11 (skip step 9).}

2. Repeat sequences 1 to 2 .

\section{COMMENTARY}

\section{Background Information}

\section{Thoracic aortic aneurysm}

Thoracic aortic aneurysms (TAA) and pseudoaneurysms can have several etiologies including atherosclerosis, cystic medial degeneration, trauma, infection, connective tissue disorders, and vasculitis (Figure A12.2.1). The majority of TAA can be attributed to atherosclerosis, which typically involves the descending aorta, although, any of the possible etiologies for TAA can also involve the ascending aorta (Figure A12.2.2). The normal ascending aorta should be $<3.0 \mathrm{~cm}$ in diameter and the descending aorta should be $<2.5 \mathrm{~cm}$. Generally, the risk of rupture is low if the aortic aneurysm is $<5 \mathrm{~cm}$ in diameter (Hirose et al., 1992). Aneurysms are usually monitored until they reach $6 \mathrm{~cm}$ in diameter, although the threshold for surgery is highly dependent on the patient's underlying physical condition and life expectancy. MR imaging including contrast enhanced imaging can provide adequate preoperative information such as branch vessel involvement, aneurysm extent, characteristics, and location (Krinsky et al., 1997; Figure A12.2.3). Cine imaging can be used to identify the neck and source of the contained rupture in pseudoaneurysms. Cine imaging might also be included to assess the aortic valve in patients with Marfan's, Ehrler's-Danlos, and others (see UNIT A10.1, Alternate Protocol 2).

\section{Aortic aneurysms and pseudoaneurysms}

A false aneurysm, or pseudoaneurysm, involves disruption of one or more of the arterial wall layers. Pseudoaneurysms are often described as saccular with blood generally contained by the surrounding tissue or the outer adventitia. The neck of the pseudoaneurysm can be variable in size. Although trauma is the most common etiology of thoracic aortic pseudoaneurysms, they can also arise as complications from cardiac surgery concerning valve replacements. Within the thorax or abdomen pseudoaneurysms can form subsequent to certain vascular surgical procedures such as anastomotic sites. Pseudoaneurysms can become secondarily infected or be the result of infection in the post-operative period. The lumen of pseudoaneurysms may contain patent to-and-from flow or be completely thrombosed and calcified. Surgery is advocated for most pseudoaneurysms regardless of their size.

An abdominal aortic aneurysm (AAA) is described as dilation of the abdominal aorta with a wall-to-wall diameter $>3 \mathrm{~cm}$ or localized dilation of the vessel by $>50 \%$ of its normal diameter (Johnston et al., 1991). The incidence bypass grafting, particularly at cannulation or
ALTERNATE PROTOCOL
Acquired Aortic Disease

A12.2.9 


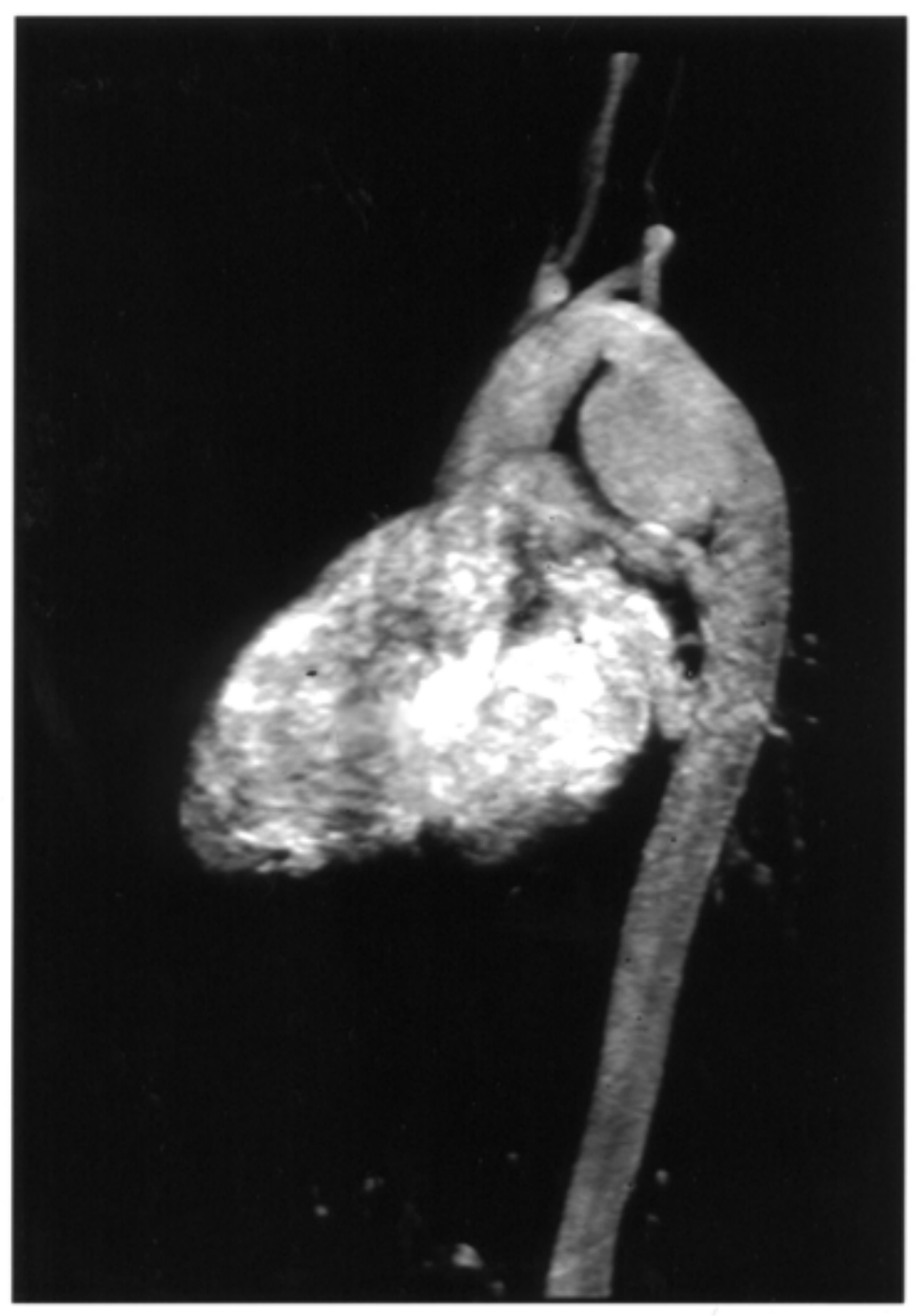

Figure A12.2.1 Pseudoaneurysm. Sagittal oblique maximum intensity projection (MIP) of the proximal aorta reveals a saccular pseudoaneurysm in the region of the isthmus.

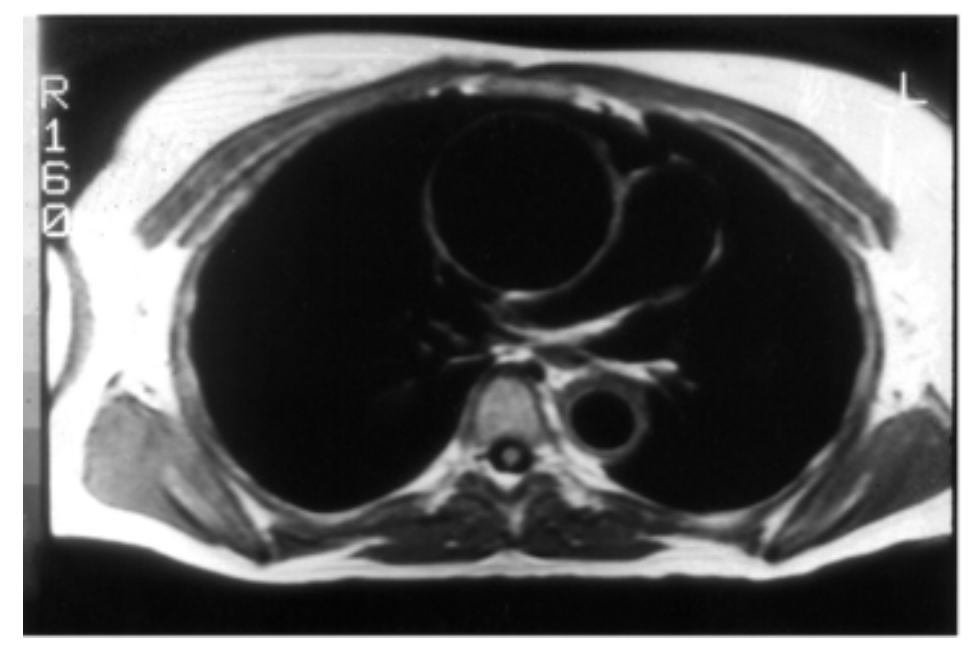

Aortic

Aneurysm and Pseudoaneurysm Assessment

Figure A12.2.2 Ascending aortic aneurysm. Transverse double inversion recovery image demonstrates a $6 \mathrm{~cm}$ aneurysm in the ascending aorta at the level of the pulmonary artery. The etiology of this aneurysm was Takayasu's Arteritis.

A12.2.10 


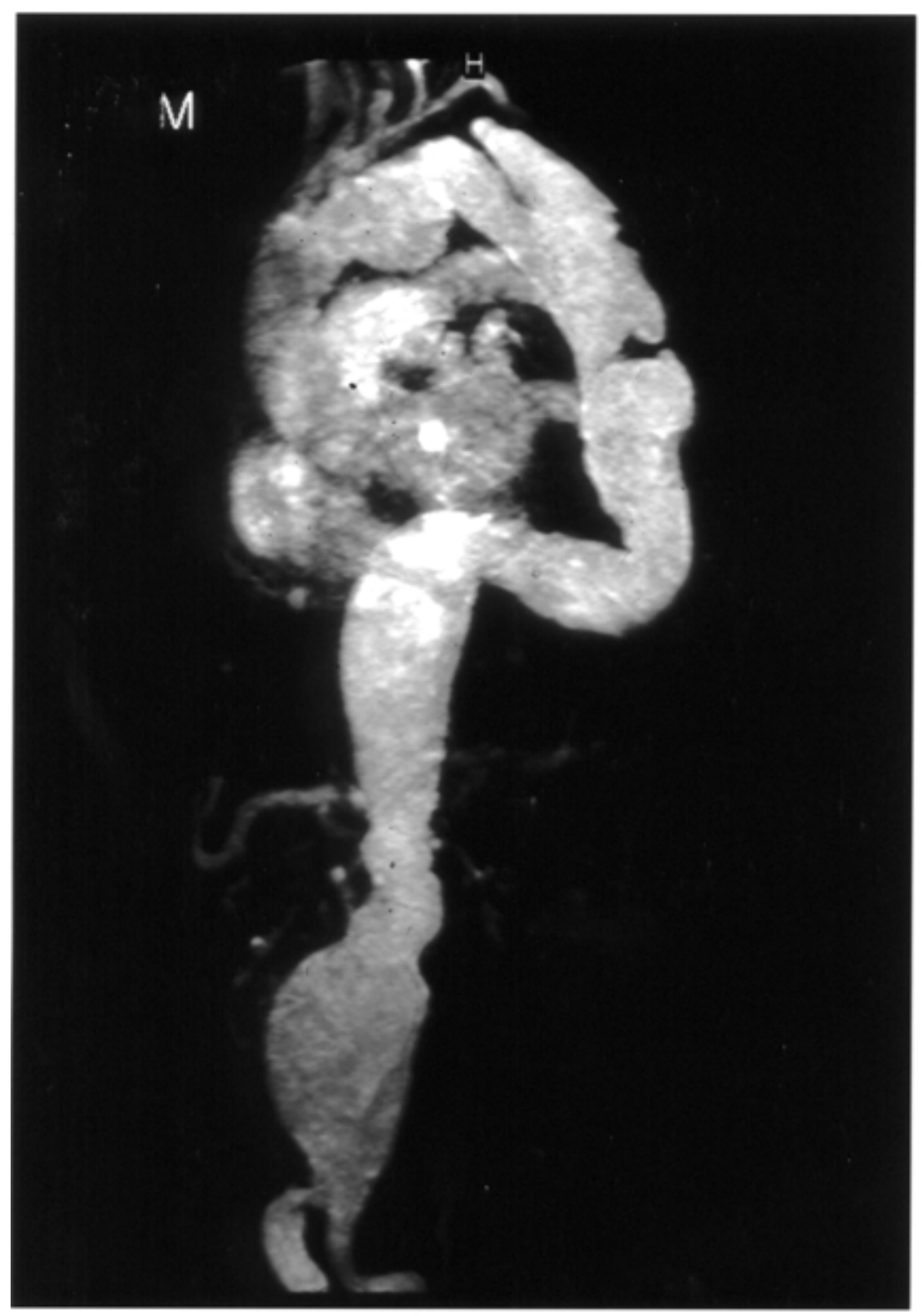

Figure A12.2.3 Penetrating ulcer. Coronal MIP image of a contrast-enhanced MRA (CEMRA) demonstrates a markedly tortuous thoracic aorta with a focal aneurysm in the aortic arch and a large penetrating ulcer in the proximal descending thoracic aorta. An infrarenal abdominal aortic aneurysm is also present.

of AAA continues to increase largely due to earlier detection and aging of the population. Most AAA's are detected incidentally. In those patients who are symptomatic, the common manifestations include abdominal pain, abdominal mass, and distal embolization.

AAA are often described as either fusiform (concentric dilation) or saccular (asymmetric dilation). Suprarenal, juxtarenal, and infrarenal are terms used to describe the location and extent of AAA. The most common cause of AAA is atherosclerosis with associated degeneration. Other less common causes include chronic dissections, infection, trauma, inflammation, and vasculitis. Although CT angiography and ultrasound have played significant roles in the detection and surveillance of AAA, contrast enhanced MRA (CEMRA) continues to emerge as a powerful tool in the preoperative and postoperative assessment of AAA (Schoenberg et al., 1999; Grist, 2000). CEMRA can provide high-resolution three-dimensional imaging without ionizing radiation or the risks associated with iodine-based radiographic contrast media. MRA provides key preoperative information regarding the proximal and distal
Acquired Aortic Disease

\section{A12.2.11}




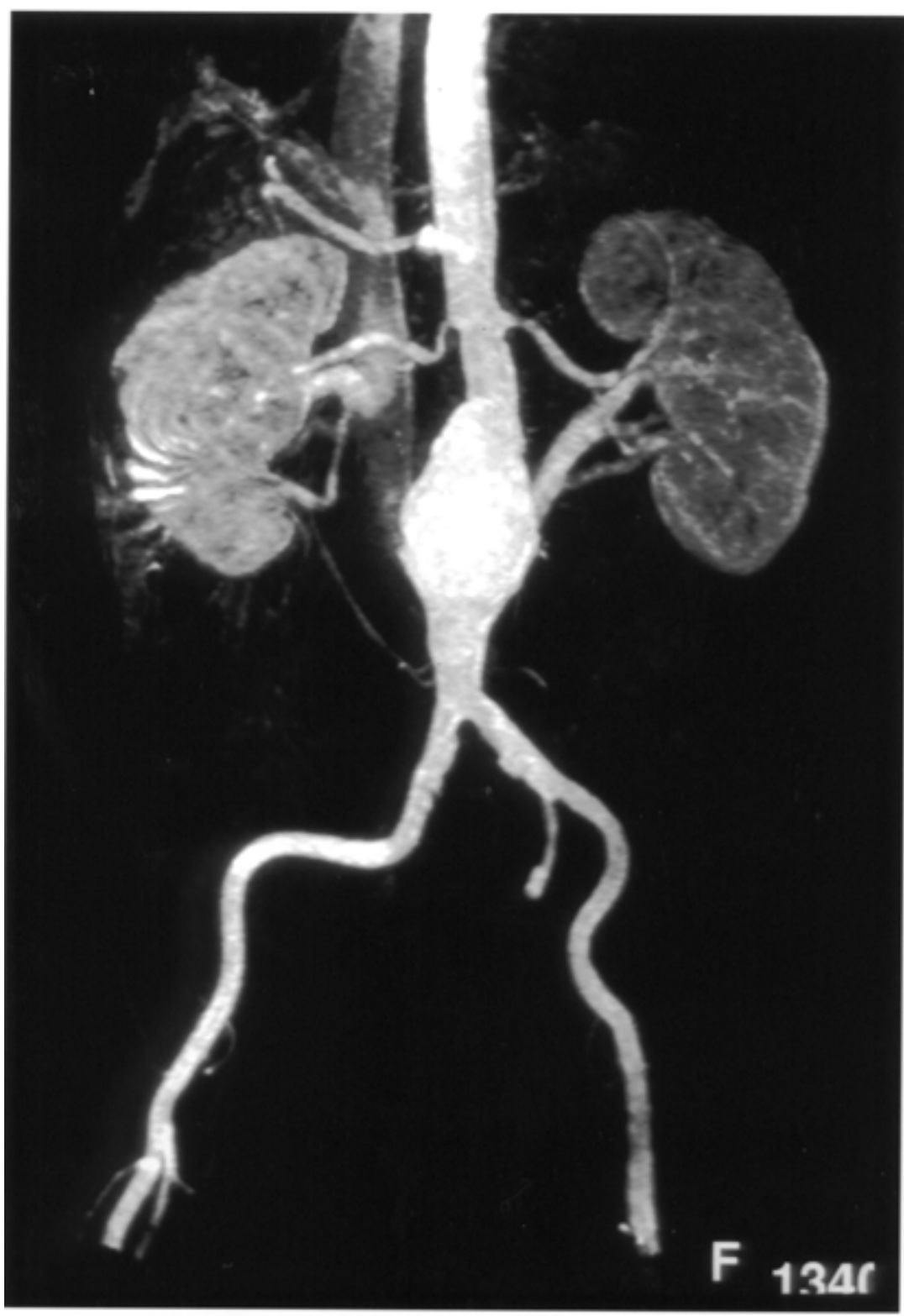

Figure A12.2.4 Abdominal aortic aneurysm with accessory right renal artery. Coronal MIP image of the abdominal aorta demonstrates an infrarenal aneurysm without extension into the common iliac arteries. A subtle small accessory right renal artery is also seen arising from the distal abdominal aorta.

extent of the aneurysm (Figure A12.2.4). It also provides information concerning the number and patency of the renal arteries, patency of the mesenteric arteries, presence of an inflammatory aneurysm, and the presence of obstructive iliofemoral arterial disease (Figure A12.2.5). The presence of such disease or variants, as well as location and extent of disease, affects surgical approach and technique. Precise assessment of the infrarenal neck and the status of the iliac vessels is even more important now with the advent of percutanous aortic stent-grafts for treatment of AAA.
Most vascular surgeons agree that aneurysms $<4 \mathrm{~cm}$ in diameter should be monitored and that aneurysms $>5 \mathrm{~cm}$ should be repaired (Crawford and Hess, 1989). Intervention on AAA in the 4 to $5 \mathrm{~cm}$ range depends on the patient's underlying medical condition and expected life-expectancy, as well as whether the AAA is increasing in size on serial imaging studies. MRI can also be useful in the postoperative evaluation for complications such as anastomotic pseudoaneurysm formation and aortoenteric fistula. CEMRA should be useful for follow-up of stent-grafts for potential leaks, although this will require additional study.
Aneurysm and Pseudoaneurysm Assessment 


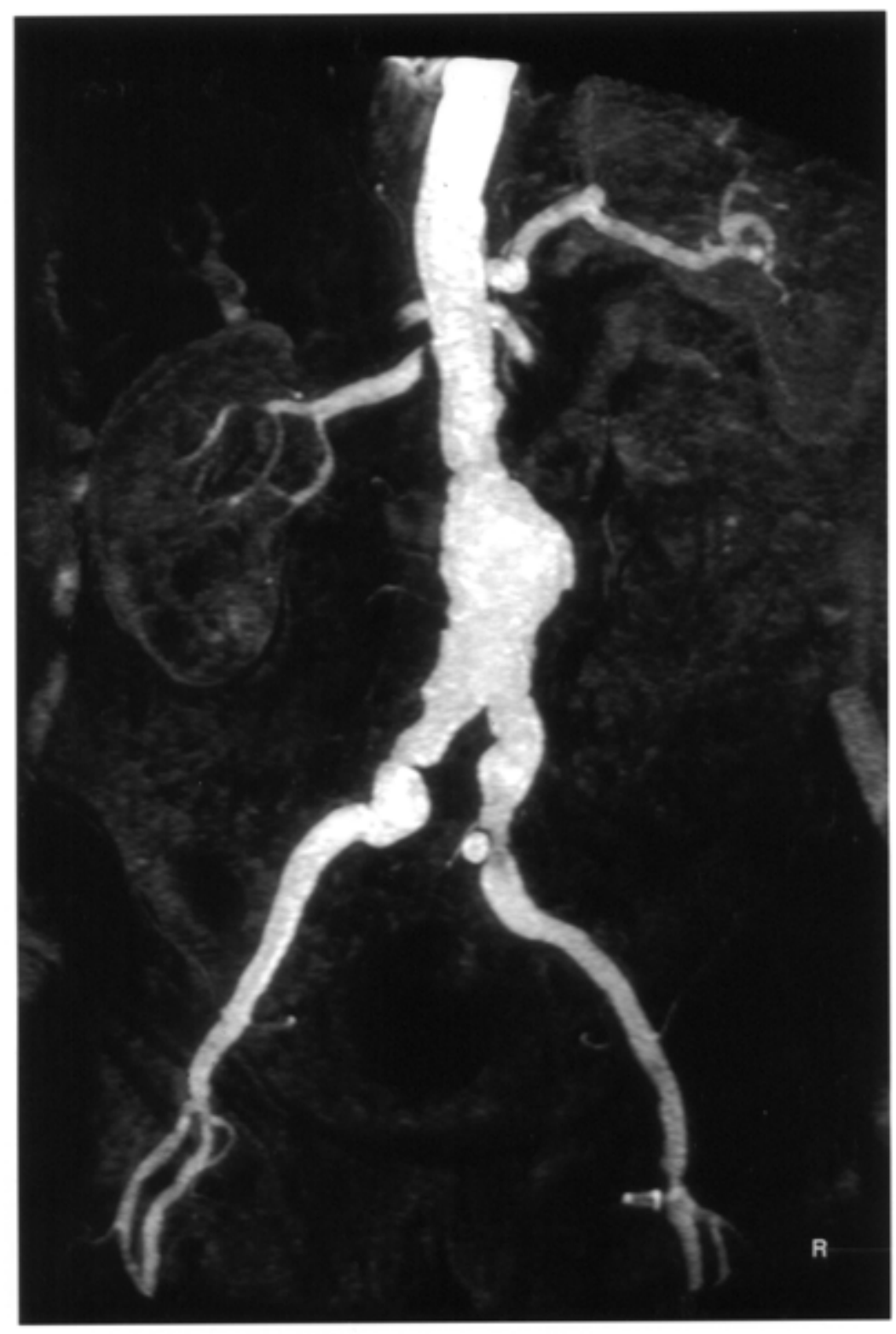

Figure A12.2.5 Coronal CEMRA of the distal aorta reveals a fusiform distal abdominal aortic aneurysm with extension into the common iliac arteries.

\section{Critical Parameters and Troubleshooting}

Volume coverage is critical, especially when the iliac arteries are tortuous. This can be remedied by increasing the slice thickness as needed.

The MIPs can overestimate stenosis. Therefore, it is important to review the source images to obtain precise measurements and determine the degree of stenosis, especially in the renal arteries and iliac arteries.

The timing of the contrast bolus is a crucial part of MRA. Ringing artifacts can occur if the central $k$-space data are acquired during the rising portion of the contrast-enhancement curve during arterial enhancement. With auto- mated detection, patients with slow flow may require up to a $10-\mathrm{sec}$ delay between detection of bolus and scan initiation. Routinely obtaining a second phase helps. Venous phase imaging is generally important if there is a problem with arterial phase timing (not uncommon in aneurysms) and for seeing false lumen of dissection.

\section{Anticipated Results}

The goals of MR imaging of the thoracic and abdominal aorta in patients with suspected aneurysms are detection, surveillance, preoperative and postoperative assessment. With new advances in technique and the emerging role of
Acquired Aortic Disease

A12.2.13 
contrast enhanced MRA, the sensitivity of this valuable imaging tool approaches that of conventional angiography. Certainly the lack of ionizing radiation and nephrotoxicity provides an advantage over CT angiography. The ability to identify the extent of the aneurysm, branch vessel involvement, and congenital variants clearly provides more information than sonography. The major role of CEMRA in the evaluation of TAA and AAA is likely in the preoperative assessment. For example, extension of the aneurysm into the iliac vessels would require placement of a bifurcated graft. Identifying the superior most extent of an TAA or AAA and its relationship to the left subclavian artery origin or the renal artery origin is important because their involvement alters the surgical approach.

\section{Literature Cited}

Crawford, E.S. and Hess, K.R. 1989. Abdominal aortic aneurysm. N. Engl. J. Med. 321:10401042.

Grist, T. 2000. MRA of the abdominal aorta and lower extremities. J. Mag. Reson. Imaging 11:32-43.

Hirose, Y., Hamada, S., Takamiya, M., Imakita, S., Naito, H., and Nishimura, T. 1992. Aortic aneurysm: Growth rates measured by CT. Radiology 185:249-252.

Johnston, K.W., Rutherford, R.B., Tilson, M.D., Shah, D.M., Hollier, L., and Stanley, J.C. 1991. Suggested standards for reporting on aortic aneurysms. J. Vasc. Surg. 13:452-458.

Krinsky, G.A., Rofsky, N.M., DeCorato, D.R., Weinreb, J.C., Earls, J.P., Flyer, M.A., Galloway, A.C., and Colvin, S.B. 1997. Thoracic aorta: Comparison of gadolinium-enhanced three-dimensional MR angiography with conventional MR imaging. Radiology 202:183-193.

Schoenberg, S.O., Wunsch, C., Knopp, M.V., Essig, M., Hawighorst, H., Laub, G., Prince, M.R.,
Allenberg, J.R., and Van Kaick, G. 1999. Abdominal aortic aneurysm. Detection of multilevel vascular pathology by time-resolved multiphase 3D gadolinium MR angiography: Initial report. Invest. Radiol. 34:648-659.

Shellock, F.G. 1996. Pocket Guide to MR Procedures and Metallic Objects. Lippincott-Raven, Philadelphia.

\section{Key References}

Fenlon, H.M. and Yucel, E.K. 1999. Advances in abdominal, aortic, and peripheral contrast-enhanced MR angiography. In Magnetic Resonance Imaging Clinics of North America: New Techniques in Body MR Imaging. W. B. Sauders Company, Philadelphia.

Reviews basic principles and new MRA techniques in the evaluation of the aorta and peripheral arteries.

Prince, M.R., Grist, T.M., and Debatin, J.K., 1999. 3D Contrast MR Angiography. 2nd Ed. Springer-Verlag, New York.

Provides practical information on the technical aspects and clinical applications of MR angiography.

Yucel, E.K., Anderson, C.M., Edelman, R.R., Grist, T.M., Baum, R.A., Manning, W.J., Culebras, A., and Pearce, W. 1999. Magnetic resonance angiography: Update on applications for extracranial arteries (AHA Scientific Statement). Circulation 100:2284-2301.

The American Heart Association Science Advisory and Coordinating Committee reviews the current clinical applications and provides recommendations for the use of MRA in the extracranial vessels. Potential future clinical applications for MRA are also presented.

Contributed by Maricela Contreras, Rajesh S. Amin, and E. Kent Yucel

Brigham \& Women's Hospital

Boston, Massachusetts
Aortic

Aneurysm and Pseudoaneurysm Assessment

A12.2.14 